\title{
Technologien des Selbst im Alltag
}

\section{Eine Einführung in relational-materielle Perspektiven}

JÖRG NIEWÖHNER \& MICHALIS KONTOPODIS

Mit Beginn der 1990er Jahre kommt eine Entwicklung zu ihrer vollen Entfaltung, die den Geistes- und Sozialwissenschaften insofern neues Terrain eröffnet, als sie die wechselseitige Beziehung zwischen dem Menschlichen und dem Nicht-Menschlichen symmetrisch konzipiert. Paradigmatisch für diese Entwicklung stehen zwei Monographien: Die Neuerfindung der Natur: Primaten, Cyborgs und Frauen von Donna Haraway (1995a) und Wir sind nie modern gewesen von Bruno Latour (1995). Während Haraway vor allem eine Kritik der dichotomen Konzeptionalisierung von Natur und Kultur im Blick hat und sich dabei auf anthropologische wie wissenschaftshistorische Arbeiten stützt (z.B. Knorr-Cetina 1984, Hagner et al. 1994; Rheinberger 1992; Rheinberger/Hagner 1993), steht für Latour zunächst die kategorische Unterscheidung zwischen dem ,Sozialen“ und verschiedenen Formen von Technik im Vordergrund. Beide jedoch sehen die Lösung ihres Problems letztlich in Praxiskonzepten, die menschliche und nichtmenschliche Akteure bzw. Handlungsträgerschaft symmetrisch konzipieren: materiell-semiotische Praxis und Akteur-Netzwerke. Seit diesen wegweisenden Monographien sind die wechselseitigen Beziehungen zwischen dem Menschlichen und dem Nicht-Menschlichen zunehmend und auf verschiedenste Art und Weise symmetrisch untersucht worden (Beck 1997; Haraway 1995b; Kneer et al. 2008; Latour 1996; 2001; 2007; Rheinberger et al. 1997).

Nach den diskursiven, bildlichen und performativen ,turns‘ (Wulf 2004) etabliert sich somit durch diese hier skizzierte Entwicklung eine Perspektive, die häufig als, relational-materiell` bezeichnet wird 
(Law/Hassard 1999). Diese Perspektive stellt sowohl die Konstitution von Subjekt und Objekt als Entitäten in Frage als auch die herkömmliche Verteilung von Handlungsträgerschaft auf eben diese Entitäten. Der relationale Materialismus markiert eine prozessuale Perspektive, die Handlungsträgerschaft nicht a priori lediglich menschlichen Subjekten zuordnet, sondern prinzipiell symmetrisch auf menschliche und nicht-menschliche Akteure verteilt und als jeweils in spezifischen Praxen aktualisiert konzipiert. ${ }^{1}$ Der Soziologe und Wissenschaftsforscher John Law benennt zwei Grundannahmen eines solchen relationalen Materialismus: (1) Im Rekurs auf Semiotik wird zunächst davon ausgegangen, dass die Formen und Attribute aller Entitäten immer durch die Beziehungen zu anderen Entitäten produziert werden. Sie sind relational zu verstehen. Diese Relationalität der Entitäten wird nicht nur auf linguistische Phänomene angewandt, sondern im Sinne einer Semiotik der Materialität auf jede Art von Phänomen ausgedehnt. ${ }^{2}$ (2) Diese Form der Relationalität verweist auch auf die zentrale Stellung von Performativität ${ }^{3}$. Nicht nur gewinnen die Entitäten ihre Form und Attribute durch Beziehungen, sondern sie werden performiert in, durch und mit diesen Beziehungen (Law 1999). Phänomene existieren also in relevanter Weise überhaupt nur so, wie sie in spezifischen Praxisformen aufeinander bezogen sind bzw. sich beziehen. Diese Perspektive richtet den Blick auf das Unfertige, das Unsichere und die Reversibilität von alltäglichen Ordnungen. Im Prinzip kann jede Be-

1 Als Vorfahren dieser ,Wende" in den Sozialwissenschaften können die psychologischen Arbeiten von Vygotsky (1978/1930) und Leont'ev (1978) wie auch die philosophischen Positionen von James (1904) und Dewey (Hickman 2004) gesehen werden. Zu einem erweiterten Kreis von Vorläufern müssen ebenfalls naturphilosophische und prozessontologische Positionen gezählt werden, die gegen Ende des 19. und am Anfang des 20. Jahrhunderts versucht haben, etablierte Dualismus zu überwinden; insbesondere zwischen Natur und Kultur (z.B. Buchanan 2008; Koutroufinis 2007; Latour 2007; Stengers 2002).

2 Hierin zeigt sich die Nähe des relationalen Materialismus zu anthropologischen Theoriesträngen, die davon ausgehen, dass Phänomene vor allem auch dadurch Bedeutung erlangen, dass sie durch (Alltags)Praxen zueinander in Beziehung gesetzt werden.

$3 \mathrm{Zu}$ einer plausiblen Unterscheidung von Performativität und Performanz siehe Schuegraf (2008). 
ziehung immer auch anders aktualisiert werden. Daher fragt der relationale Materialismus vor allem nach den kontingenzreduzierenden Ordnungsmechanismen, die spezifische Konstellationen stabilisieren und bestimmte Dualismen sichtbar machen: Wie wird Beständigkeit erreicht ? $^{4}$

Das ,Selbst', um das es in den Beiträgen dieses Bandes vor allem geht, stellt aus relational-materieller Perspektive ebenfalls keine Entität dar. Vielmehr wird jedes ,Selbst' in einem Netz von Beziehungen immer wieder neu produziert. Im Gegensatz zu vielen gängigen Selbstkonzepten der Sozialforschung hat es daher weder eine Zeitlichkeit in sich (wie etwa in den Identitätstheorien von Freud oder von Erikson: Cole 1971; Erikson/Hügel 1966), noch lässt es sich als klar definierbares System begreifen, welches zu einem Minimum von Tätigkeit tendiert, wie beispielsweise in der Luhmannschen Systemtheorie (Luhmann 1987). Das Selbst stabilisiert sich nicht nur in Beziehungen zu anderen Subjektivitäten (Mead 1968), ${ }^{5}$ sondern auch in Beziehungen zu Objektivitäten (Law/Hassard 1999; Law/Moser 2003). Parasiten, Rhizome, Akteur-Netzwerke und Cyborgs sind dabei Metaphern, die das Selbst als kontingente und mannigfaltig verschränkte Prozesse charakterisieren (Deleuze/Guattari 1980/1987; Haraway 1995c; 1997; Kneer et al. 2008; Latour 1999; Serres 1980/1982). Diese Metaphern sind vor allem dazu genutzt worden, das ständige materiell-semiotische ,Hin-und-her-Schieben“ der Grenzen des Körpers zu problematisieren. Foucault hat hierfür den Begriff Technologien des Selbst entwickelt, der nicht nur die diskursiven Praxen der Konstitution des Selbst bezeichnet, sondern auch ihre materiellen Aspekte: Die Überwachung des Selbst in staatlichen Institutionen setzt eine bestimmte Architektur voraus (Foucault/Seitter 1976) und das Schreiben von Tagebüchern ist nicht ohne das Objekt ,Tagebuch` zu denken (Martin et al. 1993). Besonders

4 Diese ontologisch-epistemologischen Grundannahmen rekurrieren auf die Semiotik von Peirce und die Prozessphilosophie von Whitehead vom Anfang des 20. Jahrhunderts und markieren damit einen Bruch mit der kontinentalen ,Substanzphilosophie', die bisher weite Teile der Sozialforschung untermauert hat (Pape 1988; Whitehead 1978/1929).

5 Weiterführend hierzu sind Ansätze aus der Sozial- und Kulturpsychologie (Gergen 1996; 2002; Hermans/Kempen 1993; Simão/Valsiner 2007) wie auch aus der Soziologie (Goffman 1971). 
anschaulich wird diese notwendige Verschränkung von semiotischen und materiellen Aspekten des Selbst in den zahlreichen Analysen von ,Biopolitik' (Foucault 1982; 1986; Lemke 2008; Rose 2006).

In den letzten etwa zehn Jahren sind überwiegend im englischsprachigen Raum eine Reihe von Arbeiten entstanden, die Körperpraxen z.B. der Reproduktion, des Altwerdens, des Krank- und Gesundseins oder der Behinderung aus relational-materieller Sicht erforschen. ${ }^{6}$ In der deutschsprachigen Fachliteratur setzt diese Entwicklung mit einer gewissen Verzögerung ein (z.B. Beck 2005; Knecht 2006; Niewöhner et al. 2008). Der vorliegende Band nimmt diese Anfänge auf und entwickelt sie mit Blick auf medizinische und technologische Eingriffe in Körper und körpernahe Praxen (Warneken 2006).

\section{BEITRÄGE ZU EINER RELATIONAL-MATERIELLEN PERspeKtive IN DIESEM BAND}

Relationaler Materialismus hat zumindest zwei wichtige methodologische Konsequenzen: Erstens folgt er einem symmetrischen Zugang und zweitens wird besonderes Augenmerk auf Praxis gerichtet. Der symmetrische Zugang ermöglicht eine Unterwanderung der kategorischen Trennung zwischen Subjekt und Objekt, die viele als kennzeichnend für westliche Modernen identifiziert haben (Latour 1995). Als solche bestimmt diese Trennung vor allem wissens- und technologiebasierte Untersuchungsfelder. Die Fähigkeit, diese Dualismen von Subjekt und Objekt oder allgemeiner von Natur und Kultur zu reproduzieren, gilt als Ausweis einer modernen, d.h. vernünftigen, reflektierten und produktiven Haltung und Organisation.

6 Siehe z.B. zur Analyse von technoscience Asdal et al. (2007); zu AIDS und der Arbeit von Patientenorganisationen Epstein (1996); zur Entwicklung der Biowissenschaften Franklin/Lock (2003); zu Hirntod und Organtransplantation im Kulturvergleich Lock (2002); allgemeiner zu kritischen medizinanthropologischen Perspektiven auf Körper und körpernahe Praxen Lock/Farquhar (2007); zum weiblichen Körper in der Reproduktionsmedizin Martin (1987); zur Produktion multipler Körper in klinischen Praxen Mol (2002) oder zur Praxis der Amniozentese und ihren sozialen Auswirkungen Rapp (1999). 
Viele Ansätze aus einer sozialkonstruktivistischen Tradition laufen Gefahr, diese Dualismen unter umgekehrten Vorzeichen zu reproduzieren. Sie implizieren ein präformiertes Soziales. Der relationale Materialismus hingegen versteht materiell-semiotische Praxis als die grundlegende analytische Einheit und untersucht diese mittels ethnographischer Methoden. Diese Perspektive bietet eine wichtige Alternative gerade in Feldern mit einem hohen Reflektionsgrad (Marcus 2008), da sie auf eine genaue Beschreibung von Ereignissen und Aktivitäten abzielt, statt auf die Beschreibungen dieser Ereignisse durch die beteiligten Akteure (Mol 2002). Praxiographisches Arbeiten eröffnet damit erstens eine wichtige Möglichkeit, den vielen Unstimmigkeiten, Variationen und Widersprüchen von moderner Wissensarbeit auf die Spur zu kommen. Zweitens ermöglicht es, der Materialität der Praxen in der sozialwissenschaftlichen Analyse gerecht $\mathrm{zu}$ werden und die Eigenschaften konkreter Objekte zu berücksichtigen (Mol 2002; 2008; Mol/Law 2004; Moser 2008). Da eine solche Analyse Wissen aus anderen Fachdisziplinen wie z.B. den Lebenswissenschaften voraussetzt, und außerdem Erfahrungswissen von TechnologieNutzer_innen oder von Patient_innen miteinbeziehen soll, kommen häufig auch ethnographische und Experteninterviews als Forschungswerkzeug zum Einsatz. Eine relational-materialielle Perspektive ermöglicht es den Sozialwissenschaften, in einen Dialog mit den Natur-, Lebens- und Technikwissenschaften zu treten und durch Alltagsethnographien zur Herstellung von transdisziplinären Wissensbeständen beizutragen. Exemplarisch dafür sind in diesem Band sowohl der Beitrag von Stefanie Zimmer als auch der Beitrag von Stefan Reinsch, die sich beide explizit der Lösung offener Fragen anderer Disziplinen und Praxisfelder widmen.

Zimmers Beitrag Die materielle Realität der Virtuellen Treppe: Ethnographische Gang-Analyse von Gesunden und SchlaganfallPatienten in der Reharobotik wendet sich der Problematik der Gangrehabilitation nach einem Schlaganfall zu. Die Analysen ihrer einjährigen Feldforschung in der Reharobotik zu den Gangmaschinen GT1, HapticWalker und Lokomat wie auch ihre eigenen KörperErfahrungen weisen darauf hin, dass der Gang im Sinne des symmetrischen Ansatzes der Akteur-Netzwerk-Theorie als punktualisiertes Netzwerk begriffen werden kann. Mit Hilfe des praxiographischen Ansatzes des „Doing Body“ (Mol/Law 2004) 
untersucht Zimmer, inwiefern die Muskelaktivität beim Treppensteigen mit dem HapticWalker Aufschluss über den TherapieErfolg geben kann. An einem konkreten Fall, bei dem ein Gesunder auf der Maschine gehen lernt, wird aus einer vergleichenden Perspektive argumentiert, dass das aktualisierte Muster nicht einer Treppe als Objekt, sondern einer personenspezifischen Technik entspricht, es jedoch der Artikulation verkörperten Wissens bedarf, um die Mannigfaltigkeit sowie den relationalen und kontingenten Charakter der emergenten Ontologie des Ganges zu begreifen.

In seinem Beitrag Lernen zu mukoviszidieren: Translationen von Jugendlichen und Therapeuten bei der Stabilisierung einer therapeutischen Beziehung widmet sich Reinsch der Thematik des Lebens mit Mukoviszidose. Auf der Basis der Soziologie der Translation beschreibt er, wie die Herstellung und Stabilisierung einer therapeutischen Beziehung zwischen Patient und Ärztin gezielt die Grenzen zwischen Krankenhauswelt und Lebenswelt problematisiert und bedingt auflöst. Durch diese Auflösung wird Leben mit Mukoviszidose zu einer medizinischen Lebensform. Diese Perspektive deutet ebenfalls an, wie eine behandlerzentrierte Konzeption „Compliance mit einem Therapieregime“ - zugunsten einer partnerschaftlichen Konzeption - „Verhandlung der Therapie“ verändert werden kann.

Der Frage nach der Veränderung und Erweiterung des menschlichen Körpers durch technische und medizinische Interventionen widmet sich auch der Beitrag von Nora Walther. Vor dem Hintergrund medizinanthropologischer und kulturhistorischer Frauenforschung untersucht Walther im Kapitel Transformation durch ein kontrazeptives Hormonimplantat: Mit implantierter Kontinuität zu imaginierter Neutralität hormonelle Verhütung durch das ,Stäbchenimplantat‘. Durch die kontinuierliche Abgabe von Etonogestrel, einem Hormon aus der Gruppe der Gestagene, wirkt dieses Implantat verhütend. In Interviews mit Nutzerinnen, gynäkologischen Fachärzt_innen und Informationen von Pharmafirmen und Internetforen untersucht die Autorin verschiedene Metaphern über Hormone z.B. als ,selbst agierende“ Instanzen oder als ,Haushalt‘. Sie analysiert die Wirkung des Implantats auf die Selbstwahrnehmung der Nutzerinnen und entwirft das Konzept der ,imaginierten Neutralität‘, um eine spezifische Form der Geschlechtsidentität zu bezeichnen. 
Der Prozess, den Zimmer, Reinsch und Walther charakterisieren, ist vor allem als materielles Werden $\mathrm{zu}$ verstehen. Es geht nicht vornehmlich um postmoderne Identitäten oder nicht-lineare biographische Erzählungen (siehe z.B. Allolio-Näcke et al. 2005; Brockmeier 1999), sondern um materiell-semiotische Praxen (Haraway 1997), die das Selbst in Beziehung zu Werkzeugen, Technologien, Räumlichkeiten und Zeitlichkeiten stellen und es dabei reterritorialisieren (Deleuze/Guattari 1980/1992). Dinge werden dabei konzipiert als ,,a stabilizing and destabilizing process of iterative intraactivity“ wie Karen Barad (2003: 821f.) schreibt.

Der vermittelnden Rolle von Technologie in Reterritorialisierungsprozessen widmet sich auch Markus Quetsch allerdings nicht in einem medizinischen Kontext. Vielmehr untersucht er in seinem Text Körper mit Profilen:gayromeo.com: eine DatingPlattform als Mediator und Quasi-Matchmaker? die Online-Datingund Social-Web-Plattform gayromeo.com als Vermittler spezifischer Prozesse des Kennenlernens und Kontakte-Pflegens. Durch die Analyse von Interviews mit Nutzern und die teilnehmende Beobachtung von Nutzung stellt Quetsch dar, wie Nutzer mit ihrem Online-Profil eine neue Handlungseinheit bilden, die sowohl auf der Ebene neuer Fähigkeiten als auch auf der Ebene neuer (Selbst)Wahrnehmung zum Tragen kommt. Besonderes Augenmerk wird hier auf die Plattform an sich gerichtet, die als ,Quasi-Objekt' eine aktive Rolle bei der Kommunikation und Profilbildung spielt (Serres 1982/1980), d.h. auf spezifische Art und Weise stabilisierend wirkt.

Dieser Frage nach der Stabilisierung heterogener Netzwerke - nun wieder in medizinischen Kontexten - wird auch in den Beiträgen von Lydia-Maria Ouart und Denny Chakkalakal nachgegangen. In ihrem Kapitel „Umrechnen auf täglich.“ Wie in Pflegegutachten Zahlen entstehen beschreibt Ouart anhand von teilnehmender Beobachtung, wie die Minutenangaben in den Begutachtungen des Medizinischen Dienstes der Krankenversicherung (MDK) zur Pflegebedürftigkeit von Patient_innen zustande kommen. Die Analyse deutet darauf hin, dass die Festlegung von Pflegestufen keine mechanische Implementierung des Sozialgesetzes ist. Stattdessen ist es für die Entstehung eines Gutachtens notwendig, dass eine Vielzahl von Aktanten in der Begutachtung kooperiert. Die Gutachterinnen des MDK bringen außerdem ein spezifisches Erfahrungswissen in die Gutachten ein und transformieren die vielschichtigen Aspekte des Alltagslebens der 
Begutachteten durch drei Mechanismen - Fokussieren, Sequenzieren und Übersetzen - in zählbare Angaben.

Anschließend daran untersucht Chakkalakal in seiner ethnographischen Studie Gesund, bewusst und richtig: Ethnographie einer ambulanten kardiologischen Rehabilitation den Versuch der Lebensstilveränderung innerhalb einer ambulanten kardiologischen Rehabilitation. Neben einer Beschreibung der Implementierung und Stabilisierung einer Gesundheitsvorstellung über die Begriffe Risiko und Wahrscheinlichkeit geht es um die Produktion eines inhärent kranken Körpers über routinisierte Gesundheitspraxen wie Blutdruckmessen bzw. -beobachten. Patient_innen müssen innerhalb dieses Gesundheitskonzepts zu risikobewussten Personen und damit zu Risikomanagern ihres Körpers werden. Letztlich wird versucht, die Reha als Ort einer Selbstaktivierung von Patient_innen zu verstehen, zum einen in der Überwindung ihrer Krankheit und zum anderen im Kampf gegen routinisiertes Alltagsverhalten.

Die Frage nach der Entstehung neuer Formen von Selbstinterpretation und Alltagspraxis durch medizinisches Wissen greift der Beitrag von Mirjam Staub auf: Medizinische Praxis in einem Zentrum für Brustkrebserkrankungen: Somatische Individualität und Biosozialität als Ergebnis von Differenzierungspraxen im Spannungsfeld zwischen Individualisierung und Kollektivbildung. Die Autorin untersucht hier die Frage, wie sich Selbstinterpretationen und soziale Alltagspraxen von Brustkrebspatientinnen als Folge medizinischer Praxis verändern. Ihre explorative Forschung in einem deutschen Zentrum für Brustkrebserkrankungen kombiniert teilnehmende Beobachtung und qualitative Interviews mit Patientinnen und Vertreter_innen der Klinik. Das Forschungsmaterial weist darauf hin, dass Individualisierungen einerseits als Folge biomedizinischen Wissens und der medizinischen Praxis zu betrachten sind. Sie werden andererseits von den Patientinnen gleichzeitig als Distanzierungen gegenüber der Krankheit praktiziert und führen dadurch zu neuen Gemeinschaftsbildungen. Staub leistet hiermit einen kritischen Beitrag zur Diskussion über Biosozialität (Rabinow 1992) und weist darauf hin, dass somatische Individualität und Biosozialität als Folge dieser medizinischen Praxis in einem Spannungsverhältnis stehen, so dass es nicht zu einer Kollektivbildung über die gemeinsame Diagnose Brustkrebs kommt.

Alle diese Beiträge verdeutlichen ebenfalls, dass Materie im Rahmen des relationalen Materialismus relational-prozessual konzipiert 
wird. Es geht um ,Prozesse“ und ,Genealogien` und nicht um ,Evolution" wie in naturwissenschaftlichen Epistemologien - auch nicht um ,Logik der Geschichte' wie im Historischen Materialismus ${ }^{7}$. Die Analysen von Deleuze sowie die von Foucault, Braidotti, Haraway und anderen weisen darauf hin, dass die relational-materielle Annäherung keine ,neue Mode' darstellt, sondern ein äußerst politisches Unternehmen ist (Barad 2003; Braidotti 1994; Law 2004). Dabei wird nicht in erster Linie Hierarchie oder Widerstand thematisiert, wie in einer Tradition der kritischen Theorie verbreitet (Horkheimer/Adorno 1969), sondern vor allem Normalisierung bzw. Homogenisierung oder auch Differenz, Flucht und Brüche (Braidotti 1994; Deleuze 1992; Foucault 1972; Haraway 2003). In den Vordergrund rücken ,Politics of Life" und neue Formen von Sozialität, die das moderne Verständnis von Politik grundsätzlich in Frage stellen (Agamben 1998; Rabinow 1992; Rose 2006).

\section{Ausblick}

Das vorliegende Buch versucht, auf die in Psychologie und Sozialforschung etablierten Konzepte des ,Selbst' ein etwas anderes Licht zu werfen. Dabei wird besonderes Augenmerk auf Materialitäten und deren Beziehungen gerichtet. Deleuze und Guattari folgend könnte man auf der Grundlage der in diesem Buch präsentierten Forschungen und theoretischen Analysen sagen, dass das ,Selbst‘ als Rhizom zu konzipieren ist:

„Ein Rhizom ist als unterirdischer Strang grundsätzlich verschieden von großen und kleinen Wurzeln. Zwiebel- und Knollengewächse sind Rhizome

7 Deleuze folgend entsteht aus der Perspektive der Dialektik nichts radikal Neues, sondern nur Rekombinationen vom Alten (Deleuze 1994/1968). In dem Sinne ist die Hegelsche Philosophie wie auch der Historische Materialismus ,Substanzphilosophie‘. Stattdessen definiert Deleuze in seiner Philosophie alle Entitäten relational d.h. in Bezug auf andere Entitäten. Wenn sich eine Beziehung zwischen Entitäten verändert, entstehen neue Entitäten. Es gibt nichts, das als Substanz in der Zeit existiert - die Zeit als solche wird auch relational geschaffen und prozessual performiert oder enacted (Kontopodis 2009; Latour 1994). 
[...]. Das Rhizom selber kann die unterschiedlichsten Formen annehmen, von der verästelten Ausbreitung in alle Richtungen an der Oberfläche bis zur Verdichtung in Zwiebeln und Knollen. [...] Ein Rhizom kann an jeder beliebigen Stelle gebrochen und zerstört werden, es wuchert entlang seiner eigenen oder anderen Linien weiter. [...] Jedes Rhizom enthält Segmentierungslinien, nach denen es geschichtet ist, territorialisiert, organisiert, bezeichnet, zugeordnet etc.; aber auch Deterritorialisierungslinien, an denen es unaufhaltsam flieht [...]. Es kann nicht wie im Baummodell entschieden werden, welches Element das grundlegendste, der ,Stamm“ ist, von dem alle anderen abhängen. Je nach Betrachtungsperspektive kann das Zentrum eines Rhizoms überall und nirgends sein. Seine einzelnen Punkte können und sollen untereinander verbunden werden (,Konnexion"). Unterschiedlichste Sachverhalte können miteinander in Verbindung treten (,Heterogenität'). Jedes Rhizom ist nicht in sich abgeschlossen, sondern existiert einzig in seinen internen Beziehungsfeldern und in den Verhältnissen, die es zu externen anderen Beziehungsfeldern hat. Aus all diesen Eigenschaften folgt, dass das Rhizom keine abstrakte Einheit als ideelle Vorstellung sein kann, sondern eher der Name für einen Prozess“. (Deleuze/Guattari 1980/1992)

In der Gesundheitsforschung und -politik, in der Psychiatrie, aber auch in den Minderheitenstudien und in Bereichen wie Pädagogik oder Migrationspolitik ist relationaler Materialismus nicht nur mit neuen theoretischen Sichtweisen, sondern auch mit neuen Praxen verbunden (Bowker/Star 1999; Kontopodis 2007; 2008; Papadopoulos et al. 2008). Äußerst signifikant sind die Anwendungen von relational-materiellen Ansätze auch im Bereich der so genannten ,politischen Ökologie“ (Haraway 2008; Latour 2001). Brüche, dezentrale Organisation und Heterogenität rücken gegenüber modernen Ordnungen in den Vordergrund. Wir betrachten dieses Buch als einen Beitrag zu einer Diskussion über diese Themen im deutschsprachigen Raum. Wir sind uns bewusst, dass die Texte in ihrer Gesamtheit viele Fragen offen und andere gänzlich unberührt lassen. Doch zugleich stellen sie viele bekannte Fragen auf ungewohnte Art und Weise, und wir hoffen, dass diese neuen Problematisierungen Wissen darstellen, um das herum unsere Leserschaft Neues wird erfinden können (Strathern 2002). 


\section{LITERATUR}

Agamben, Giorgio (1998): »Homo sacer: Sovereign power and bare life «. Stanford: Stanford University Press.

Allolio-Näcke, L./Kalscheuer, B./Manzeschke, A. (2005): »Differenzen anders denken: Bausteine zu einer Kulturtheorie der Transdifferenz $«$. Frankfurt a. M. und New York: Campus.

Asdal, K./Brenna, B./Moser, I. (2007): »Technoscience: The politics of interventions «. Oslo: Unipub.

Barad, Karen (2003): »Posthumanist Performativity: Toward an Understanding of How Matter Comes to Matter«. Signs: Journal of Women in Cultur and Society 28, S. 801-831.

Beck, Stefan (1997): »Umgang mit Technik: Kulturelle Praxen und kulturwissenschaftliche Forschungskonzepte «. Berlin: Akademie.

Beck, Stefan (Hg.) (2005): »Alt sein - Entwerfen - Erfahren: Ethnografische Erkundungen in Lebenswelten alter Menschen«. Berlin: Panama

Bowker, Geoffrey/Star, Susan L. (1999): »Sorting things out: Classification and its consequences «. Cambridge, MA.: MIT Press.

Braidotti, Rosi (1994): »Nomadic Subjects: Embodiment and Sexual Difference in Contemporary Feminist Theory«. New York: Columbia University Press.

Brockmeier, Jens (1999): »Erinnerung, Identität und autobiographischer Prozess«. Journal für Psychologie 7, S. 22-42.

Buchanan, Brett (2008): »Onto-ethologies: The animal environments of Uexküll, Heidegger, Merleau-Ponty, and Deleuze«. Albany: SUNY Press.

Cole, J. Preston (1971): »The problematic self in Kierkegaard and Freud «. New Haven: Yale University Press.

Deleuze, Gilles (1992): »Postscript on the Societies of Control«. October 59, S. 3-7.

Deleuze, Gilles (1994/1968): »Difference and repetition«. London: Athlone.

Deleuze, Gilles/Guattari, Félix (1980/1987): »A Thousand Plateaus: Capitalism and Schizophrenia«. Minneapolis: University of Minnesota Press.

Deleuze, Gilles/Guattari, Félix (1980/1992): »Tausend Plateaus: Kapitalismus und Schizophrenie«. Berlin: Merve. 
Epstein, Steven (1996): »Impure science: AIDS, activism, and the politics of knowledge «. Berkeley: University of California Press.

Erikson, Erik H./Hügel, Käte (1966): »Identität und Lebenszyklus: drei Aufsätze«. Frankfurt a. M.: Suhrkamp.

Foucault, Michel (1972): »The Archaeology of Knowledge and the Discourse on Language«. New York: Harper and Colophon.

Foucault, Michel (1982): »Afterword: The Subject and Power«. In: H.L. Dreyfus et al. (Hg.): Michel Foucault: Beyond Structuralism and Hermeneutics. Chicago: University of Chicago Press, S. 208225.

Foucault, Michel/Seitter, Walter (1976): »Überwachen und Strafen: Die Geburt des Gefängnisses«. Frankfurt a. M.: Suhrkamp.

Franklin, Sarah/Lock, Margaret (Hg.) (2003): »Remaking Life and Death: Toward an Anthropology of the Biosciences«. Santa Fe: School of American Research Press.

Gergen, Kenneth J. (1996): »Das übersättigte Selbst: Identitätsprobleme im heutigen Leben«. Heidelberg: Auer.

Gergen, Kenneth J. (2002): »Konstruierte Wirklichkeiten: Eine Hinführung zum sozialen Konstruktionismus«. Stuttgart: Kohlhammer.

Goffman, Erving (1971): »Verhalten in sozialen Situationen: Strukturen und Regeln der Interaktion im öffentlichen Raum«. Gütersloh: Bertelsmann.

Hagner, M./Rheinberger, H.-J./Wahrig-Schmidt, B. (1994): »Objekte, Differenzen, Konjunkturen: Experimentalsysteme im historischen Kontext«. Berlin: Akademie.

Haraway, Donna (1995a): »Ein Manifest für Cyborgs: Feminismus im Streit mit den Technowissenschaften«. In: Dies.: Die Neuerfindung der Natur: Primaten, Cyborgs und Frauen, Frankfurt a. M./New York: Campus, S. 33-72.

Haraway, Donna (1995b): »Monströse Versprechen: Coyote-Geschichten zu Feminismus und Technowissenschaft «. Berlin: Argument.

Haraway, Donna (1995c): »Die Neuerfindung der Natur: Primaten, Cyborgs und Frauen«. Frankfurt a. M.: Campus.

Haraway, Donna (1997): »Modest_Witness@Second_Millennium. FemaleMan $\mathbb{C}_{-}$Meets_OncoMouse ${ }^{\mathrm{TM}} \ll$. New York: Routledge. 
Haraway, Donna (2003): »The Companion Species Manifesto: Dogs, People, and Significant Otherness «. Chicago: Prickly Paradigm Press.

Haraway, Donna (2004): »The Haraway Reader«. New York: Routledge.

Haraway, Donna (2008): »When Species Meet«. Minneapolis: University of Minnesota Press.

Hermans, Hubert J. M./Kempen, Harry J. G. (1993): »The dialogical self: Meaning as movement «. San Diego: Academic Press.

Hickman, Larry A. (2004): »John Dewey: Zwischen Pragmatismus und Konstruktivismus«. Münster: Waxmann.

Horkheimer, Max/Adorno, Theodor (1969): »Dialektik der Aufklärung «. Frankfurt a. M.: Fischer.

James, William (1904): »The principles of psychology«. New York: Holt.

Knecht, Michi (2006): »Zwischen Religion, Biologie und Politik: Eine kulturanthropologische Analyse der Lebensschutzbewegung in der Bundesrepublik Deutschland «. Münster: LIT.

Kneer, G./Schroer, M./Schüttpelz, E. (Hg.) (2008): »Bruno Latours Kollektive: Kontroversen zur Entgrenzung des Sozialen«. Frankfurt a. M.: Suhrkamp.

Knorr-Cetina, Karin (1984): »Die Fabrikation von Erkenntnis: Zur Anthropologie der Naturwissenschaften«. Frankfurt a. M.: Suhrkamp.

Kontopodis, Michalis (2007): »Human Development as Semiotic-material Ordering: Sketching a Relational Developmental Psychology?«. Critical Social Studies 9, S. 5-20.

Kontopodis, Michalis (2008): »The „Transitory“ Moment: On Time \& Subjectivity in Contemporary Art and Art and Dance Therapy«. In: Ruth Hampe et al. (Hg.): Grenzüberschreitungen - Bewusstseinswandel und Gesundheitshandeln. Berlin: Frank \& Timme, S. 459467.

Kontopodis, Michalis (2009): »Editorial: Time. Matter. Multiplicity«. Memory Studies 2, S. 5-10.

Koutroufinis, Spyridon (Hg.) (2007): »Prozesse des Lebendigen: Zur Aktualität der Naturphilosophie A. N. Whiteheads«. Freiburg und München: Karl Alber. 
Latour, Bruno (1994): »Les objets ont-ils une histoire? Rencontre de Pasteur et de Whitehead dans un bain d'acide lactique«. In: Isabelle Stengers (Hg.): L'effet Whitehead, Paris: Vrin, S. 197-217.

Latour, Bruno (1995): »Wir sind nie modern gewesen: Versuch einer symmetrischen Anthropologie«. Berlin: Akademie.

Latour, Bruno (1996): »Der Berliner Schlüssel: Erkundungen eines Liebhabers der Wissenschaften«. Berlin: Akademie-Verlag.

Latour, Bruno (1999): »Pandora's Hope: Essays on the Reality of Science Studies«. Cambridge, MA: Harvard University Press.

Latour, Bruno (2001): »Das Parlament der Dinge: Für eine politische Ökologie«. Frankfurt a. M.: Suhrkamp.

Latour, Bruno (2007): »Eine neue Soziologie für eine neue Gesellschaft: Einführung in die Akteur-Netzwerk-Theorie«. Frankfurt a. M.: Suhrkamp.

Law, John (1999): »After ANT: Complexity, naming and topology«. In: John Law et al. (Hg.): Actor network theory and after. Oxford and Malden, MA: Blackwell, S. 1-15.

Law, John (2004): »After Method: Mess in Social Science Research«. London: Routledge.

Law, John/Hassard, John (1999): »Actor network theory and after«. Oxford and Malden, MA: Blackwell.

Law, John/Moser, Ingunn (2003): »Managing, Subjectivities and Desires«. Centre for Science Studies, Lancaster University, http://www.comp.lancs.ac.uk/sociology/papers/Law-MoserManaging-Subjectivities-Desires.pdf.

Lemke, Thomas (2008): »Gouvernementalität und Biopolitik«. Wiesbaden: VS Verlag.

Leont'ev, Aleksei N. (1978): »Activity, consciousness, and personality«. Englewood Cliffs, NJ: Prentice and Hall.

Lock, Margaret (2002): »Twice dead: Organ transplants and the reinvention of death «. Berkeley: University of California Press.

Lock, Margaret/Farquhar, Judith (2007): »Beyond the body proper: Reading the anthropology of material life«. Durham: Duke University Press.

Lock, M./Young, A./Cambrosio, A. (2000): »Living and working with the new medical technologies: Intersections of inquiry«. Cambridge and New York: Cambridge University Press.

Luhmann, Niklas (1987): »Soziale Systeme: Grundriß einer allgemeinen Theorie «. Frankfurt a. M.: Suhrkamp. 
Marcus, George (2008): »The end(s) of ethnography: Social/cultural anthropology's signature form of producing knowledge in transition «. Cultural Anthropology 24, S. 1-14.

Martin, Emily (1987): »The woman in the body: A cultural analysis of reproduction «. Boston: Beacon Press.

Martin, L. H./Gutman, H./Hutton, P. H./Foucault, M. (1993): »Technologien des Selbst «. Frankfurt a. M.: Fischer.

Mead, George H. (1968): »Geist, Identität und Gesellschaft aus der Sicht des Sozialbehaviorismus«. Frankfurt a. M.: Suhrkamp.

Mol, Annemarie (2002): »The body multiple: Ontology in medical practice«. Durham: Duke University Press.

Mol, Annemarie. (2008): »I Eat an Apple: On Theorizing Subjectivities«. Subjectivity 22, S. 28-37.

Mol, Annemarie/Law, John (2004): »Embodied Action, Enacted Bodies: The Example of Hypoglycaemia«. Body Society 10, S. 43-62.

Moser, Ingunn (2008): »Making Alzheimer's Disease Matter: Enacting, Interfering and Doing Politics of Nature «. Geoforum 39, S. 98-110.

Niewöhner, J./Kehl, C./Beck, S. (2008): »Wie geht Kultur unter die Haut? Emergente Praxen an der Schnittstelle von Medizin, Lebensund Sozialwissenschaft«. Bielefeld: transcript.

Papadopoulos, D./Stephenson, N./Tsianos, V. (2008): »Escape routes: Control and subversion in the twenty-first century«. London: Pluto Press.

Pape, Helmut (Hg.) (1988): »Charles Peirce: Naturordnung und Zeichenprozess: Schriften über Semiotik und Naturphilosophie«. Aachen: Alano Rader

Rabinow, Paul (1992): »Artificiality and Enlightment: From Sociobiology to Biosociality«. In: Jonathan Crary/Sanford Kwinter (Hg.): Incorporations. New York: Zone, S. 234-252.

Rapp, Rayna (1999): »Testing women, testing the fetus: The social impact of amniocentesis in America«. New York: Routledge.

Rheinberger, Hans-Jörg (1992): »Experiment, Differenz, Schrift: Zur Geschichte der epistemischen Dinge«. Marburg: Basilisken.

Rheinberger, Hans-Jörg/Hagner, Michael (1993): Die Experimentalisierung des Lebens: Experimentalsysteme in den biologischen Wissenschaften 1850/1950, Berlin: Akademie.

Rheinberger, H.-J./Hagner, M./Wahrig-Schmidt, B. (1997): »Räume des Wissens: Repräsentation, Codierung, Spur«. Berlin: Akademie. 
Rose, Nikolas (2006): »The politics of life itself: Biomedicine, power, and subjectivity in the twenty-first century«. Princeton, NJ: Princeton University Press.

Schuegraf, Martina (2008): »Medienkonvergenz und Subjektbildung. Mediale Interaktionen am Beispiel von Musikfernsehen und Internet«. Wiesbaden: VS Verlag für Sozialwissenschaften.

Serres, Michel (1982/1980): »The parasite«. Baltimore and Maryland: Johns Hopkins University Press.

Simão, Livia M./Valsiner, Jaan (2007): »Otherness in question: Labyrinths of the self«. Charlotte, N.C.: Information Age Pub.

Stengers, Isabelle. (2002): »Penser avec Whitehead: Une libre et sauvage création de concepts«. Paris: Seuil.

Strathern, Marilyn (2002): »Not giving the game away«. In: A. Gingrich et al. (Hg.), Anthropology by Comparison, London: Routledge, S. xiii-xvii.

Vygotsky, Lev S. (1978/1930): »Tool and Symbol in Child Development«. In: Lev Semënovic Vygotsky: Mind in Society: The Development of Higher Psychological Processes. Cambridge, MA: Harvard University Press, S. 19-30.

Warneken, Bernd J. (2006): Die Ethnographie popularer Kulturen: Eine Einführung, Wien, Köln und Weimar: Böhlau.

Whitehead, Alfred N. (1978/1929): »Process and Reality: An Essay in Cosmology«. New York: Free Press.

Wulf, Christoph (2004): »Anthropologie: Geschichte, Kultur, Philosophie«. Hamburg: Rowohlt. 